\title{
The Impact of Infective Immigrants and Self Isolation on the Dynamics and Spread of Covid-19 Pandemic: A Mathematical Modeling Study
}

\author{
Molalegn Ayana ${ }^{1, *}$, Tsige Hailegiorgis ${ }^{2}$, Kassahun Getnet ${ }^{3}$ \\ ${ }^{1}$ Department of Mathematics, Dilla College of Teacher Education, Dilla, Ethiopia \\ ${ }^{2}$ Department of Biology, Kotebe Metropolitan University, Addis Ababa, Ethiopia \\ ${ }^{3}$ Department of Mathematics, Hawassa University, Hawassa, Ethiopia \\ Email address: \\ molaayana@gmail.com (M. Ayana), tsigehg@gmail.com (T. Hailegiorgis), Kassaget@gmail.com (K. Getnet) \\ ${ }^{*}$ Corresponding author
}

\section{To cite this article:}

Molalegn Ayana, Tsige Hailegiorgis, Kassahun Getnet. The Impact of Infective Immigrants and Self Isolation on the Dynamics and Spread of Covid-19 Pandemic: A Mathematical Modeling Study. Pure and Applied Mathematics Journal. Vol. 9, No. 6, 2020, pp. $109-117$. doi: 10.11648/j.pamj.20200906.12

Received: August 23, 2020; Accepted: September 18, 2020; Published: November 23, 2020

\begin{abstract}
The COVID-19 pandemic is considered as the biggest global threat worldwide because of millions of confirmed infections, accompanied by hundred thousand deaths over the world. WHO is working with its networks of researchers and other experts to coordinate global work on surveillance, epidemiology, modeling, diagnostics, clinical care and treatment, and other ways to identify, manage the disease and limit onward transmission. Mathematical modeling has become an important tool in analyzing the epidemiological characteristics of infectious diseases. The present study describes the transmission pathways in the infection dynamics, and emphasizes the role of exposed (probably asymptomatic infected) and infected immigrants and the impact of self isolation techniques in the transmission and spread of covid-19 with no home to home check up to develop a mathematical model and show the impact of infected immigrants and self isolation on the dynamics and spread of covid-19. In our model we study the epidemic patterns of Covid-19, from a mathematical modeling perspective. The present model is developed making some reasonable modifications in the corresponding epidemic SCR model by considering symptomatic and asymptomatic infective immigrants as well as self isolation measures. Our numerical results indicate that the corona virus infection would remain pandemic, unless the responsible body takes Self isolation measure and intervention programs and introducing home to home check up of covid-19 to reduce the transmission of the disease from asymptomatic infected (exposed) individual to the susceptible individual. Among the model parameters the exposed and infected self isolation rate and exposed (probably asymptomatic infected) immigration rate are very sensitive parameters for the spread of the virus. Disease free equilibrium point is found and endemic equilibrium state is identified. It is shown that the disease free equilibrium point is locally and globally asymptotically stable if $\mathrm{R}_{0}<1$, and unstable if it is $\mathrm{R}_{0}>1$. Simulation study is conducted using MATLAB ode45.
\end{abstract}

Keywords: Self Isolation, Home to Home Check Up, Asymptomatic Infected, Symptomatic Infected, Corona Virus Pandemic, Covid-19

\section{Introduction}

The outbreak of the Corona virus COVID-19 has taken the lives of thousands worldwide and locked out many countries and regions, with yet unpredictable global consequences [1]. Novel corona virus the third zoonotic virus emerging in the this century, after the severe acute respiratory syndrome corona virus (SARS-CoV) in 2002 that spread to 37 countries and the Middle East respiratory syndrome corona virus (MERSCoV) in 2012 that spread to 27 countries [2].

The human corona virus Covid -19 of the century emerged in December 2019, with a cluster of patients with connections to Huanan South China Seafood Market in Wuhan, Hubei Province, China. Similar to severe acute 
respiratory syndrome corona virus (SARS-CoV) and Middle East respiratory syndrome corona virus (MERS-CoV) infections, patients exhibited symptoms of viral pneumonia including fever, difficulty in breathing, and bilateral lung infiltration in the most severe cases [2, 9, 19]. Another typical symptoms of COVID-19 infection include dry cough, fatigue, and severe cases, patients have included mild cases needing supportive care to severe cases requiring extracorporeal membrane oxygenation and Some people also develop non respiratory symptoms such as nausea, vomiting, and diarrhea similar to those caused by SARS-CoV and MERS-CoV infections [2, 8, 9]. During previous outbreaks due to other coronavirus (Middle-East Respiratory Syndrome (MERS) and Severe Acute Respiratory Syndrome (SARS)) emerged as a new human coronavirus in June 2012 [2, 8], human to human transmission occurred through droplets, contact, suggesting that the transmission mode of the Covid -19 can be similar [5]. On January 30, the World Health Organization (WHO) formally declared the outbreak of novel coronavirus a Global Public Health Emergency of International Concern (pandemic) [1, 2, 18$]$.

WHO takes deferent measures to give response for covid19 like identify, isolate and care for patients early, including providing optimized care for infected patients, address crucial unknowns and about clinical severity, extent of transmission and infection, treatment options, and accelerate the development of diagnostics, therapeutics minimize social and economic impact through multisectoral partnerships [5]. WHO is working with its networks of researchers and other experts to coordinate global work on surveillance, epidemiology, modeling, diagnostics, clinical care and treatment, and other ways to identify, manage the disease and limit onward transmission [5, 18]. Till August 15, 2020there are no antiviral drugs or vaccines currently available, clinical evidence shows that the incubation period of this disease ranges from 2 to 14 days, during this period of time, infected individuals may not develop any symptoms and may not be aware of their infection, yet they are capable of transmitting the disease to other people [2].

The COVID-19 pandemic is considered as the biggest global threat worldwide because of millions of confirmed infections, accompanied by hundred thousand deaths over the world [14, 18]. Notice, by 15 August, 2020, report more than 23 million confirmed cumulative cases with more than 756 thousand deaths worldwide.

A number of modeling studies have already been performed for the COVID-19 epidemic, and introduced a susceptible-exposed-infectious-recovered (SEIR) model to describe the transmission dynamics, and forecasted the national and global spread of the disease. The impact of migration of population on the distribution and spread of disease is required to be analyzed properly and must be understood clearly [7]. Migration and immigration of the people from one country to another country due to deferent reasons play a crucial role in the evolution and spread of infectious disease [16]. The models published thus far have not taken into account the role of exposed and infected immigrants as well as the role of self isolation from the virus in the transmission of COVID-19.

The main objective of the present study is to understand the role of exposed (probably asymptomatic infected) and infected immigrants as well as the role of self isolation technique on the spread and dynamics of corona virus in the absence of home to home check up. The transmission rates in our model depend on the epidemiological status which changes with time. In particular, when the infection level is high, people would be motivated to take necessary action to reduce the contact with the infected individuals so as to protect themselves and their families, leading to a reduction of the average transmission rates. Now, not only medical and biological research but also mathematical modeling approach also cooperates and plays a crucial role to stop COVID-19 outburst. By mathematical modeling we may forecast the point of infection and finishing time of the disease. It also helps us to make proper decision about the necessary steps to restrain the spreading the diseases.

Home to home check up: is testing individuals at their home and assessing the prevalence of Covid-19 in the population and tracing the transmission. Home to home check up is used to identify asymptomatic infected individuals as well as used to manage the contacts of susceptible and asymptomatic infected individuals.

\section{Mathematical Model Formulation}

Mathematical modeling for disease transmission has been done by many deferent authors to understand the dynamical spread of disease in humans. Models for infectious disease are helpful for prevention and control of emerging infectious disease like novel Corona virus [12, 15, 18]. Mathematical modeling has become an important tool in analyzing the epidemiological characteristics of infectious diseases [12, 18, 20]. The models are crucial for the policymaker to acquire medical supplies, allocate human resources and hospital beds, and ensure the sustainability of the health system throughout the peak and duration of the epidemic [18]. Mathematical modeling plays a key role in policy making, including risk assessment and control program evaluation in reducing morbidity and mortality [15].

The spread of the disease modeled using ordinary deferential equations ODEs where humans interact and infect each other $[1,12,16]$. The major application of these equations is when the rate of change of a variable is related to other variables, as it is so in most physical and biological systems. Many powerful mathematical tools exist for the analysis and numerical solution of models based on deferential equations [1]. Many mathematical models did not consider symptomatic and asymptomatic infected immigrants and self isolation techniques on the dynamics of infectious disease [16].

Thus, a new model that combines the SEIR system for disease transmission in the human population interaction by considering exposed and infected immigrants as well as self isolation measures. For the purpose of this study the whole 
human population is classified in to four compartments: Susceptible human population $(t)$, Exposed human population (individuals who carry the virus and can infect others but have no symptoms yet) $E(t)$, Symptomatic infected human population $I(t)$ and Recovered human population $R(t)$, by considering exposed and infected immigrants as well as self isolation techniques with no home to home check up.

Susceptible human population: This susceptible compartment is neither exposed nor infected by covid- 19 virus now. But they are very sensitive likely to be affected by or having the quality of receiving the disease in future [12]. People will join susceptible compartment by natural birth or by immigration. People go out from this compartment by natural death or go to the Exposed (probably asymptomatic infected) human population. The remaining people will stay in the susceptible compartment itself. Peoples in the susceptible compartment will get infection from Covid-19 infected human population and from exposed (probably asymptomatic infected) humans population. The susceptible population is increased by recruitment rate of $\delta$.

Exposed human population: The exposed individuals who carry the virus and can infect others but have no symptoms of the virus. Without home to home check up asymptomatic infected individuals are remain in this compartment. Peoples go out from exposed compartment either to the symptomatic compartment or recovered compartment. People will join to this compartment either from susceptible or from exposed immigration.

Infected Human population: The infected humans are those who have already been infected by Covid-19 and they show the symptoms of the disease and can transfer the disease to susceptible human. Some people will join to the symptomatic infected compartment from Exposed human population after incubation and from immigration from other place. Some people of the infected human compartment will die with natural reasons and die due to Covid -19 or go to recovered class after developing immunity.

Recovered Human Population: Exposed (probably asymptomatic infected) human populations and infected human populations move to recovered class after developing immunity. And recovered human populations will die due to natural reasons. In our case recovered individuals are assumed to be developed permanent immunity and cannot get infection again.

Table 1. The model variables and their representations.

\begin{tabular}{ll}
\hline$S(t)$ & Number of susceptible human population at any time t \\
$E(t)$ & Number of exposed human population at any time $\mathrm{t}$ \\
$I(t)$ & Number of symptomatic infected human population an time t \\
$R(t)$ & Number of recovered human population at any time $\mathrm{t}$ \\
\hline
\end{tabular}

\subsection{Basic Assumption of the Model}

In is difficult to formulate mathematical model which contains all the parameters and conditions that influence the physical problems. That is why the same problems have different mathematical model formulations. Those models are formulated by considering different assumptions. The present study has been developed based on the following Assumptions:

1) There is no home to home check up, i.e. Asymptomatic infected individual's remains as at exposed compartment.

2) The model is open (human mobility is considered).

3) There is no disease induced death in the exposed class.

4) The recovered individuals are assumed to acquire permanent immunity.

5) All the newborn ones are susceptible to the virus.

6) All parametric values are positive.

7) Exposed individuals infect susceptible individuals.

8) Natural death or due to other diseases occurs in each of the compartments.

9) Population assumed to be moved from one compartment to the other.

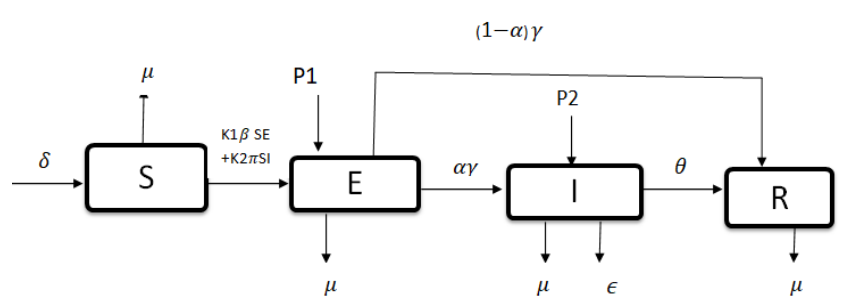

Figure 1. The compartmental structure describing Covid-19 transmission.

Considering the model assumptions and the flow diagram the dynamics and spread of Covid-19 can be described by the system of ordinary differential equations constructed in terms of notations of variables and parameters as follows:

$$
\begin{array}{r}
d S / d t=\delta-K_{1} \beta S E-K_{2} \pi S I-\mu S \\
d E / d t=K_{1} \beta S E-K_{2} \pi S I-\gamma E-\mu E+P_{1} \\
d I / d t=\alpha \gamma E-\epsilon I-\theta I-\mu I+P_{2} I \\
d R / d t=(1-\alpha) \gamma E+\theta I-\mu R
\end{array}
$$

With initial conditions $S(0)=S_{0}, E(0)=E_{0}, I(0)=$ $I_{0}, \operatorname{and} R(0)=R_{0}$.

And the total population is given by $N=S+E+I+R$

We denote $\delta$ the rate of recruitment that added to the susceptible population by birth and immigration each day and the natural death rate (in absence of Covid-19) of the population is $\mu$ per day. The parameter $\beta$ is the rate at which the susceptible human become infected by being in contact with exposed human per day; the rate that one contact between a susceptible and infected results in infection is $\pi$ per day. The parameters $\mathrm{K}_{1}$ and $\mathrm{K}_{2}$ represents self isolation measures of exposed and infected individuals respectively which is a control variable since an increase in the protection may substantially reduce the spread of covid-19 if done properly. The parameter $\gamma$ per day denotes the rate of development of clinical symptoms in exposed individuals and $\alpha$ is the mean infectious period of exposed (asymptomatic infected) individuals. The parameter $\theta$ represents the recovery rate of symptomatic infected 
individuals and $\epsilon$ represents the disease induced death rate of infected human population. The parameters $\mathrm{P}_{1}$ and $\mathrm{P}_{2}$ are the rate of exposed and infected immigrants to the respected compartments from other areas.

\subsection{Positivity of the Solution}

The general model equations of system (1-4) are to be epidemiologically meaningful and well posed; we need to prove that all the state variables are non-negative

Theorem: If $S_{0}>0, E_{0}>0, I_{0}>0$ and $R_{0}>0$ then the solutions $S(t), E(t), I(t), R(t)$ on the system of ODE given above (equation 1 to 4 ) are non-negative for all $t>0$.

Proof: To show the positivity of the solution of the dynamical system comprising the equations (1) to (4) we have to consider and verify each differential equations and show that their solution is positive. First let's consider equation (1) of the dynamical system.

$$
\begin{gathered}
d S / d t=\delta-K_{1} \beta S E-K_{2} \pi S I-\mu S \\
d S / d t \geq-K_{1} \beta S E-K_{2} \pi S I-\mu S \\
d S /_{d t} \geq\left(-K_{1} \beta E-K_{2} \pi I-\mu\right) S
\end{gathered}
$$

\subsection{Boundedness of the Solution}

The total human population $N$ is given by $N(t)=S(t)+$ $E(t)+I(t)+R(t)$ implies using equations 1 to 4 ,

$$
\begin{gathered}
d N / d t=\frac{d S}{d t}+\frac{d E}{d t}+\frac{d I}{d t}+\frac{d R}{d t} \\
d N / d t=b N-\mu N+P_{1} E+P_{2} I-\epsilon I \\
d N / d t \leq b N-\mu N+P_{1} N+P_{2} N-\epsilon N \\
\leq\left(b-\mu-\epsilon+P_{1}+P_{2}\right) N
\end{gathered}
$$

Where, $\rho=b N$ and since with out the loss of generality $E \leq N, I \leq N, R \leq N$. Therefore the solution system of human population is bounded in the region. Up on integrating and simplifying we have $N(t) \leq N_{0} e^{\int\left(b-\mu-\epsilon+P_{1}+P_{2}\right) d t}$. Equivalently

$$
\begin{gathered}
0 \leq N(t) \leq N_{0} e^{\int\left(b-\mu-\epsilon+P_{1}+P_{2}\right) d t} \text { as } t \rightarrow \infty \\
\Omega=\left\{S, E, I, R \in R^{4}: N(t) \leq N_{0} e^{\int\left(b-\mu-\epsilon+P_{1}+P_{2}\right) d t}\right\} .
\end{gathered}
$$

Up on integrating the in equality we obtain the analytic solution as:

$$
\mathrm{S}(\mathrm{t}) \geq \mathrm{S}_{0} \mathrm{e}^{\int\left(-\mathrm{K}_{1} \beta \mathrm{SE}-\mathrm{K}_{2} \pi \mathrm{SI}-\mu\right) \mathrm{dt}}
$$

Here $S_{0}$ is integral constant and represents initial population of the susceptible human compartment and $\mathrm{e}^{\int\left(-\mathrm{K}_{1} \beta S E-\mathrm{K}_{2} \pi S I-\mu\right) d t}$ is always greater than zero it is a positive quantity. Now within the limit $t \rightarrow \infty$, the analytical solution leads to $S(t)>0$. Hence the solution or the population size of the susceptible compartment $S(t)$ is always positive.

By using similar procedure we can show that all the solutions of the other compartments are all non-negative. i.e $E(t)>0, I(t)>0$ and $R(t)>0$ for all $t \geq 0$. Therefore the solutions sets $\{S(t), E(t), I(t), R(t)\}$ of the model equation 1 to 4 are all non-negative for all $t \geq 0$.

\subsection{Disease Free Equilibrium Points}

Disease-free equilibrium (DFE) points are steady-state solutions where there is no corona virus infection and the equilibrium points are obtained by setting the right hand sides of the model equations to zero $[12,16]$. Disease free equilibrium points are steady state solutions of the system of ODE in the absence of disease in the population. That is $E=0$ and $I=0$. Thus the disease free equilibrium point denoted by $E_{0}$ and given by $E_{0}=(S, E, I, R)=(\delta / \mu, 0,0,0)$.

\subsection{Basic Reproduction Number}

The basic reproduction number denoted by $R_{0}$, is defined as the number of secondary infections that result from the introduction of a single infectious individual into a completely susceptible population during its entire period of infectiousness, will be calculated by using the next generation matrix technique $[12,13,15,16]$. Basic reproductive number is discussed in order to identify influential model parameters, so with controlling parameters in it, the outbreak of the disease can be eliminated [15]. It is an important parameter in epidemiology as it sets the threshold in the study of a disease both for predicting its outbreak as well as evaluating its control strategies. Persistent and die out of the disease in a community depend on the size of the reproduction number $\mathrm{R}_{0}[13,15,16]$. If the reproductions $R_{0}$ greater than zero the disease breakout and if the reproduction number $\mathrm{R}_{0}$ less than zero, the disease dies out over the period of time $[8,12,15,16]$. We can compute the basic reproduction number using the next generation matrix approach by taking the exposed and infected compartments from the model equations (2) and (3) above. We construct the matrices $f_{i}$ and $V_{i}$ and the corresponding matrices of partial derivatives $F$ and $V$. Also we find the inverse matrix $V^{-1}$ of $V$. Finally we compute the reproduction number as the trace of the matrix $\mathrm{FV}^{-1}$. The reproduction number is the dominant eigenvalue of the matrix $F V^{-1}[7,13,12,16]$. All these matrices are computed and constructed and given

below:

$$
\begin{gathered}
f_{i}=\left[\begin{array}{c}
\mathrm{K}_{1} \beta \mathrm{SE}+\mathrm{K}_{2} \pi \mathrm{SI} \\
0
\end{array}\right] \text { and } \\
\gamma \mathrm{E}+\mu \mathrm{E}-\mathrm{P}_{1} \mathrm{E} \\
V_{i}=\left[\begin{array}{c}
\mathrm{I}+\epsilon \mathrm{I}+\mu \mathrm{I}-\alpha \gamma \mathrm{E}-\mathrm{P}_{2} \mathrm{I}
\end{array}\right] .
\end{gathered}
$$

$$
F=\partial f_{i} / \partial x_{j}=\left[\begin{array}{cc}
\mathrm{K}_{1} \beta \mathrm{S} & \mathrm{K}_{2} \pi \mathrm{S} \\
0 & 0
\end{array}\right] \text { and } \mathrm{V}=\partial \mathrm{V}_{\mathrm{i}} / \partial \mathrm{x}_{\mathrm{j}}=\left[\begin{array}{cc}
\gamma+\mu-\mathrm{P}_{1} & 0 \\
\alpha \gamma & \theta+\epsilon+\mu-\mathrm{P}_{2}
\end{array}\right]
$$


Differentiating with respect to the variables $E$ and $I$ and solving at the disease free equilibrium point gives the jacobian matrix.

$$
\begin{gathered}
V^{-1}=\left[\begin{array}{cc}
\frac{1}{\gamma+\mu-\mathrm{P}_{1}} & 0 \\
\frac{\alpha \gamma}{\left(\gamma+\mu-\mathrm{P}_{1}\right)\left(\theta+\epsilon+\mu-\mathrm{P}_{2}\right)} & \frac{1}{\theta+\epsilon+\mu-\mathrm{P}_{2}}
\end{array}\right], \text { and } \\
F V^{-1}=\left[\begin{array}{cc}
\mu \frac{\mathrm{K}_{1} \beta \delta}{\left(\gamma+\mu-\mathrm{P}_{1}\right)}+\frac{\mathrm{K}_{2} \pi \alpha \gamma \delta}{\left.\mu\left(\gamma+\mu-\mathrm{P}_{1}\right) \theta+\epsilon+\mu-\mathrm{P}_{2}\right)} & \frac{\mathrm{K}_{2} \pi \delta}{\mu\left(\theta+\epsilon+\mu-\mathrm{P}_{2}\right)} \\
0 & 0
\end{array}\right] .
\end{gathered}
$$

Also the reproductive number is the spectral radius of the matrix $\mathrm{FV}^{-1}$, obtained as $R_{0}=\rho\left(F V^{-1}\right)$.

The eigenvalues of the matrix are the solution of the $\mathrm{FV}^{-1}$ are characteristic equation of $\left|F V^{-1}-\lambda I\right|=0$, where $I$ is an identity matrix and $\lambda$ is the Eigen value of the matrix $F V^{-1}$. Solving this we have

$$
\lambda_{1}=0 \text { and } \lambda_{2}=\frac{\mathrm{K}_{1} \beta \delta}{\mu\left(\gamma+\mu-\mathrm{P}_{1}\right)}+\frac{\mathrm{K}_{2} \pi \alpha \gamma \delta}{\left.\mu\left(\gamma+\mu-\mathrm{P}_{1}\right) \theta+\epsilon+\mu-\mathrm{P}_{2}\right)} .
$$

Among those eigenvalues the dominant eigenvalue is $\lambda_{2}$.

$$
R_{0}=\frac{\mathrm{K}_{1} \beta \delta}{\mu\left(\gamma+\mu-\mathrm{P}_{1}\right)}+\frac{\mathrm{K}_{2} \pi \alpha \gamma \delta}{\mu\left(\gamma+\mu-\mathrm{P}_{1}\right)\left(\theta+\epsilon+\mu-\mathrm{P}_{2}\right)}
$$

Implies $R_{0}=\frac{\rho \delta}{\mu \mathrm{n}}+\frac{\phi \alpha \gamma \delta}{\mu \mathrm{nm}}$, where, $\rho=K_{1} \beta, \phi=K_{2} \pi$, $n=\mu+\gamma-P_{1}$ and $m=\mu+\theta+\epsilon-P_{2}$.

\section{Analysis of the Model}

The equilibrium points for the general model are identified and their stability analysis is made. The system exhibits two types of equilibrium point viz., disease free equilibrium points and endemic equilibrium points.

\subsection{Stability of Disease Free Equilibrium Point}

Disease-free equilibrium (DFE) points are steady-state solutions where there is novel corona virus (Covid-19) infection in the population and the equilibrium points are obtained by setting the right hand sides of the model equations (1) to (4) to zero.

Theorem 1: The disease free equilibrium point $E_{0}$ is locally asymptotically stable if and only if the reproduction number $R_{0}<1$ and unstable if $R_{0}>1[12,13,16]$.

Proof: The local stability of $E_{0}$ is then determined from the signs of the eigen values of the Jacobian matrix. At the disease-free equilibrium, $E_{0}$, the Jacobian matrix is given by:

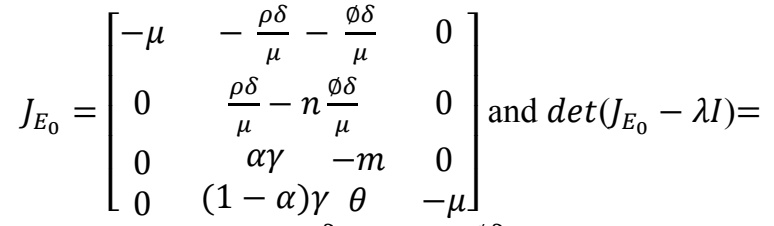

$$
\begin{aligned}
& 0,\left|\begin{array}{cccc}
-\mu-\lambda & -\frac{\rho \delta}{\mu} & -\frac{\emptyset \delta}{\mu} & 0 \\
0 & \frac{\rho \delta}{\mu}-n-\lambda & \frac{\emptyset \delta}{\mu} & 0 \\
0 & \alpha \gamma & -m-\lambda & 0 \\
0 & (1-\alpha) \gamma & \theta & -\mu-\lambda
\end{array}\right|=0
\end{aligned}
$$

The characteristic equation of the matrix $J_{E_{0}}$ is $\left|J_{E_{0}}-\lambda I\right|$ $=0$. Here $\mathrm{I}$ is an identity matrix of class $4 \times 4$ and $\lambda$ is the eigenvalue of the jacobian Matrix.

From the first and the fourth column we have two negative real roots. i.e. $\lambda_{1}=\lambda_{4}=-\mu$. The remaining roots are determined from $2 \times 2$ minor of the jacobian matrix. Let the minor is given by:

$$
A=\left[\begin{array}{c}
\frac{\rho \delta}{\mu}-n \frac{\emptyset \delta}{\mu} \\
\alpha \gamma-m
\end{array}\right]
$$

From this we have, $\operatorname{det}(A)=m n-\frac{m \delta \rho}{\mu}-\frac{\alpha \gamma \delta \phi}{\mu}=$ $\frac{\operatorname{det}(A)}{m n}=1-\frac{\rho \delta}{\mu n}+\frac{\phi \alpha \gamma \delta}{\mu n m}=1-R_{0} \quad, \quad$ from this we have $\operatorname{det}(A)>0$, if and only if $R_{0}<1$. And $\operatorname{trac}(A)=$ $\frac{\rho \delta}{\mu}-n-m, \operatorname{trac}(A)<0$ if and only if $\frac{\rho \delta}{\mu}<n+m[8,20]$.

Therefore, since the eigenvalues of $A$ are both negative when $\operatorname{det}(A)>0$, (recall that $\operatorname{trac}(A)<0)$, we conclude that $E_{0}$ is locally asymptotically stable if and only if $R_{0}<1$. When $R_{0}>1$, the matrix has a positive real eigenvalue and this means that the disease free equilibrium is unstable.

Even one can show that the disease free equilibrium point is stable if $R_{0}<1$ by using Routh Hurwitz criterion [12].

Theorem 2: The disease free equilibrium point $E_{0}$ of the system of ordinary deferential equations is globally stable in the region if $R_{0}<1[12,13,16]$.

Proof: The proof is based on using a comparison theorem. The equation of the Exposed and Infected components can be written in terms of

$$
\left[\begin{array}{l}
d E / d t \\
d I / d t
\end{array}\right]=(F-V)\left[\begin{array}{c}
E \\
I
\end{array}\right]-\left[\begin{array}{c}
0 \\
\alpha \gamma E
\end{array}\right]\left[\begin{array}{l}
d E / d t \\
d I / d t
\end{array}\right] \leq(F-V)\left[\begin{array}{c}
E \\
I
\end{array}\right]
$$

And also all the eigenvalues of the matrix $[F-V]$ have negative real parts. It follows that the system of linear differential inequalities (8) is stable whenever $R_{0}<1[16]$.

\subsection{The Endemic Equilibrium}

Endemic equilibrium point is a steady-state solution, where the disease persists in human population. This equilibrium implies that if the carrier population is present in the system, then the infection will be transmitted to the human population. Endemic equilibrium $E_{1}$ of our model is obtained by setting right hand side of the model system of equation equal to zero $[12,16]$.

The endemic equilibrium point $E_{1}$ is denoted by $E_{1}=$ $\left(S^{*}, E^{*}, I^{*}, R^{*}\right)$ 


$$
\begin{aligned}
& \delta-K_{1} \beta S E-K_{2} \pi S I-\mu S=0 \\
& K_{1} \beta S E-K_{2} \pi S I-\gamma E-\mu E+P_{1} E=0 \\
& \alpha \gamma E-\epsilon I-\theta I-\mu I+P_{2} I=0 \\
& (1-\alpha) \gamma E+\theta I-\mu R=0
\end{aligned}
$$

On solving the foregoing system of equations we get the following:

$$
\begin{gathered}
S^{*}=\frac{n m}{\rho m+\alpha \emptyset \gamma}, E^{*}=\frac{\mu m\left(R_{0}-1\right)}{\rho m+\alpha \emptyset \gamma} \\
I^{*}=\frac{\mu \alpha \gamma\left(R_{0}-1\right)}{\rho m+\alpha \emptyset \gamma}, R^{*}=\frac{\gamma\left(R_{0}-1\right)(m+\alpha \theta-\alpha m)}{\rho m+\alpha \emptyset \gamma}
\end{gathered}
$$

where, $\rho=K_{1} \beta, \phi=K_{2} \pi, n=\mu+\gamma-P_{1}$ and $m=\mu+$ $\theta+\epsilon-P_{2}$.

All the endemic equilibrium points are positive whenever the reproduction number $R_{0} \geq 1$. We apply the linearization technique in the system to determine the stability of the endemic equilibrium.

Theorem 3: The Endemic equilibrium point of system of equation the model is locally asymptotically stable if and only if the reproduction number $R_{0} \geq 1$ and unstable if $R_{0}<1$ [13].

Proof: The jacobian matrix is given by:

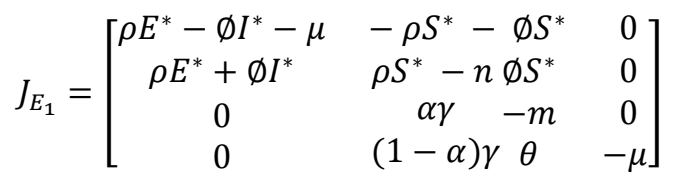

The characteristic equation of the matrix $J_{E_{1}}$ is
$\left|J_{E_{1}}-\lambda I\right|=0$. Here $I$ is an identity matrix of class $4 \times 4$ and $\lambda$ is the eigenvalue of the jacobian Matrix. From the last column we have one negative real root $\lambda_{4}=-\mu$. The remaining roots are determined from the minor of the jacobian matrix $[8,20]$. Let the minor is given by

$$
B=\left[\begin{array}{cc}
\rho E^{*}-\phi I^{*}-\mu-\lambda & -\rho S^{*}-\phi S^{*} \\
\rho E^{*}+\phi I^{*} & \rho S^{*}-n-\lambda \phi S^{*} \\
0 & \alpha \gamma-m-\lambda
\end{array}\right]
$$

Solving the determinant of the above matrix we have $\operatorname{det}\left(J_{E_{1}}-\lambda I\right)=0$ on matrix $\mathrm{B}$.

Simplifying the expression we have the expression of the form:

$$
\lambda^{3}+a_{1} \lambda^{2}+a_{2} \lambda+a_{3}=0
$$

$$
\begin{aligned}
& \text { Where, } a_{1}=\rho E^{*}+\phi I^{*}+\mu+m+n-\rho S^{*} \\
& \begin{array}{r}
a_{2}=\rho m E^{*}+\rho n E^{*}+\phi m I^{*}+\phi n I^{*}+\mu n+m n \\
-\rho \mu S^{*}-\rho m S^{*}-\alpha \gamma \phi S^{*}+\mu n
\end{array} \\
& a_{3}=\rho m n E^{*}+\phi m n I^{*}+\mu m n-\rho \mu m S^{*}-\mu \alpha \gamma \phi S^{*}
\end{aligned}
$$

Since solving the above characteristic polynomial for eigenvalues is tedious we will use the Routh-Hurwitz criterion to determine whether all roots have negative real parts and establish the stability of the system without solving the characteristic equation itself. By Routh Hurwitz criteria the determinant of Hurwitz matrix becomes positive if the following conditions hold true $a_{1}>0, a_{2}>0$ and $a_{1} a_{2}>$ $a_{3}$.

It required that all these requirements should hold true in our present model $[14,16]$. Implies.

$$
\begin{gathered}
a_{1}>0 \text { if and only if } \mu\left(R_{0}-1\right)+\mu+n+m>\rho m n / \rho m+\alpha \gamma \phi \\
a_{2}>0 \text { if and only if } \mu\left(R_{0}-1\right)(m+n)+\mu(m+n)>\rho \mu m n / \rho m+\alpha \gamma \phi \\
a_{3}=\mu m n\left(R_{0}-1\right) \\
a_{1} a_{2}=\mu R_{0}(m+n)\left[\mu\left(R_{0}-1\right)+m+n+\mu\right]-\frac{\rho \mu m n}{\rho m+\alpha \gamma \phi}\left[\mu\left(R_{0}-1\right)+n+m+\mu+R_{0}(m+n)+\rho m n / \rho m+\alpha \gamma \phi\right] \\
a_{1} a_{2}>a_{3} \text { if and only if } \mu R_{0}(m+n)\left[\mu\left(R_{0}-1\right)+m+n+\mu\right]>\frac{\rho \mu m n}{\rho m+\alpha \gamma \phi}\left[\mu\left(R_{0}-1\right)+n+m+\mu+R_{0}(m+n)+\right. \\
\rho m n / \rho m+\alpha \gamma \phi
\end{gathered}
$$

If those conditions are satisfied the roots of the characteristics equation becomes negative. Hence all roots of the characteristic polynomial are negative and this verifies that the system of equation (1) to (4) is locally asymptotically stable. Therefore the endemic equilibrium is stable if $R_{0}>1$ otherwise it is unstable $[12,13,18]$.

\section{Simulation Study of the Model}

Our numerical simulations examine the impact of infective symptomatic and asymptomatic immigrants and self isolation measures on the transmission of the Covid19 disease using MATLAB ode 45 and show the sensitive parameters on the dynamics of the disease. The main focus of the simulation study is to investigate the response of model parameters up on the covid-19 pandemic. Some of the parameter values are taken from literatures and the others are by assumptions. The parametric values are given in the following table and their unit is day. 
Table 2. Model parameters their description and values.

\begin{tabular}{llll}
\hline Parameters & Description & Values & Reference \\
\hline$\delta$ & Recruitment rate of susceptible population & 808 & Assumption \\
$\beta$ & Transmission coefficient from exposed individuals & 0.065 & {$[1,21]$} \\
$\pi$ & Transmission coefficient from infected individuals & 0.05 & {$[1]$} \\
$\alpha$ & Incubation period of exposed classes & 0.67 & {$[3,7]$} \\
$\gamma$ & Rate at which exposed people become infected I & 0.213 & {$[3,7]$} \\
$\epsilon$ & Disease induced death rate due to infected class & 0.0025 & Assumption \\
$\theta$ & Recovery rate of symptomatic infected individuals & 0.143 & {$[7]$} \\
$\mu$ & Natural death rate of individuals & 0.006 & Assumption \\
$P_{1}$ & Immigration rate fexposed individuals & Varied & Assumption \\
$P_{2}$ & Immigration rate of Infected individuals & Varied & Assumption \\
$K 1$ & Self isolation rate of exposed individuals & Varied & Assumption \\
$K 2$ & Self isolation rate of Infected individuals & Varied & Assumption \\
\hline
\end{tabular}

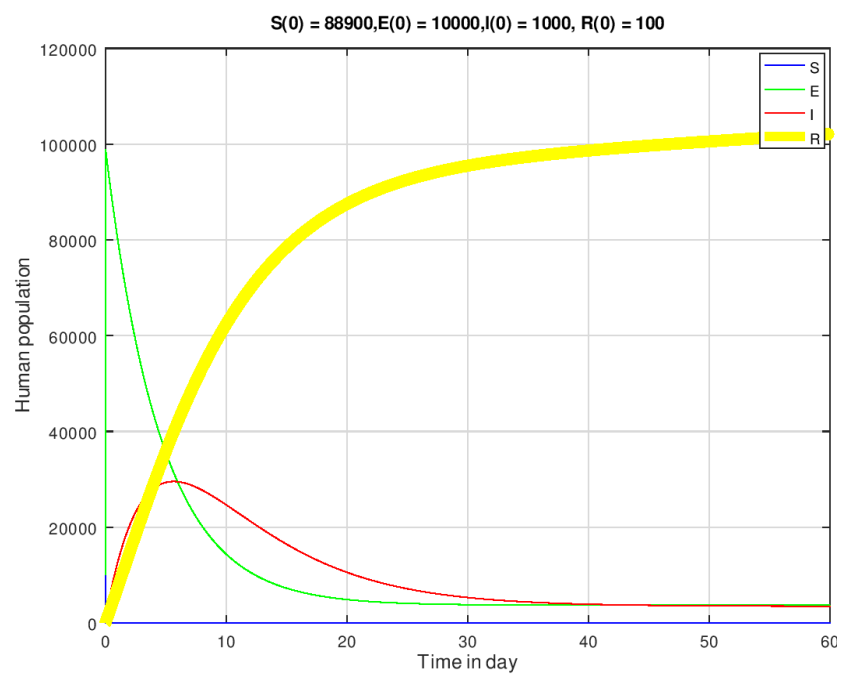

Figure 2. Numerical Simulation of the Model without considering self isolation and population mobility.

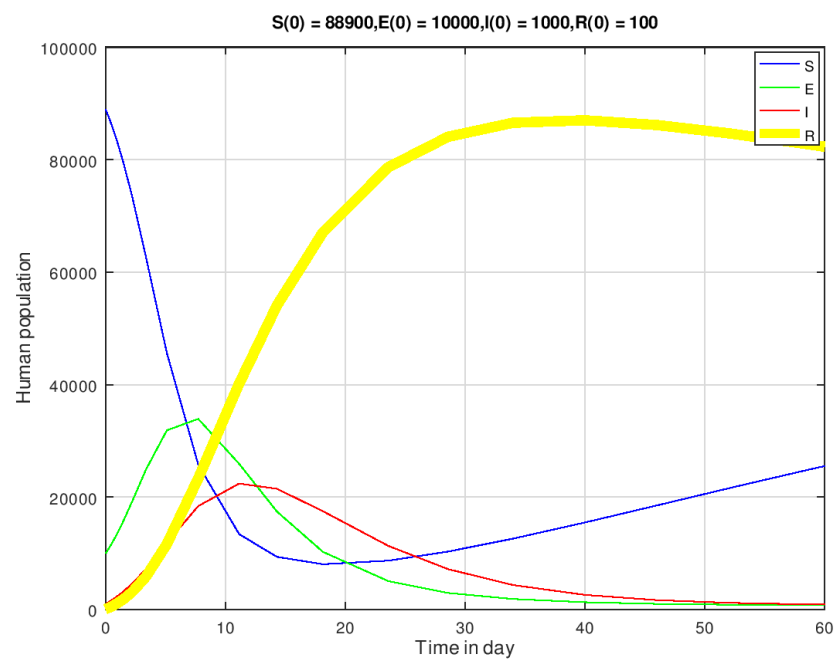

Figure 3. Numerical simulation of the Model with Self isolation measure but Immigration.

In Figure 2 the human populations are plotted vs time. As we have seen Figure 2 above with increasing time the exposed human population increases rapidly and decreases gradually, at the same time the susceptible human population decreases rapidly. The infected human population increases in the first weeks and decrease gradually with increasing time.

As we have seen from the Figure 3, human populations are plotted vs time with some self isolation measure of exposed and infected human populations. The susceptible human population decreases gradually and at the same time the exposed and infected human populations increases in the first weeks and decrease gradually. But the recovered human population increases with time because of the some self isolation measure.

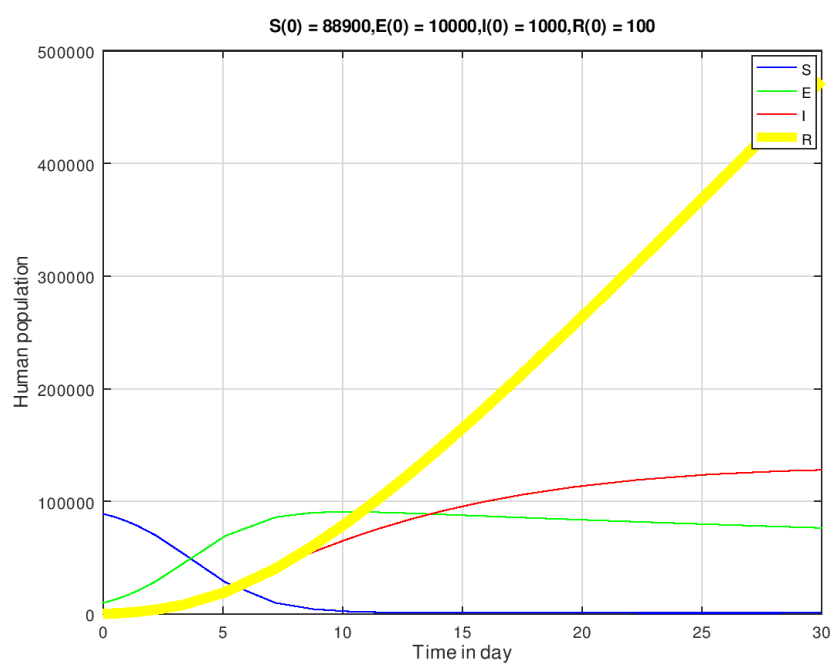

Figure 4. Numerical simulation of the Model with high Self isolation measure and low immigration rate.

In Figure 4 the Exposed and infected human population increases in the first weeks because of exposed and infected immigrants and decrease gradually with time and recovered human population increased slowly with time. At the same time the susceptible human population decreased slowly because of small self isolation measure and high immigration rate.

In Figure 5 the susceptible, exposed and infected human populations are plotted vs time. The infected human population increases rapidly because of the high immigration rate of infected human population that leads to increasing the number of infected human populations. And that is why the susceptible human population decreases rapidly because of those high exposed and infected immigrants. The exposed 
human populations initially increase rapidly because of high level of immigration rates that leads to increase the number of exposed population. The exposed and infected human populations decrease gradually because of some isolation measures.

As we have seen in Figure 6, the exposed human population and infected human population increases rapidly in the first few weeks and decrease gradually because of high exposed immigration and high infected immigration rates. But as compared with Figure 5 the exposed human population increased in the first few weeks and decreases gradually because of less exposed immigration rate. Those exposed and infected immigrant's leads to new infection of susceptible human population and that is why the susceptible human population decreases rapidly.

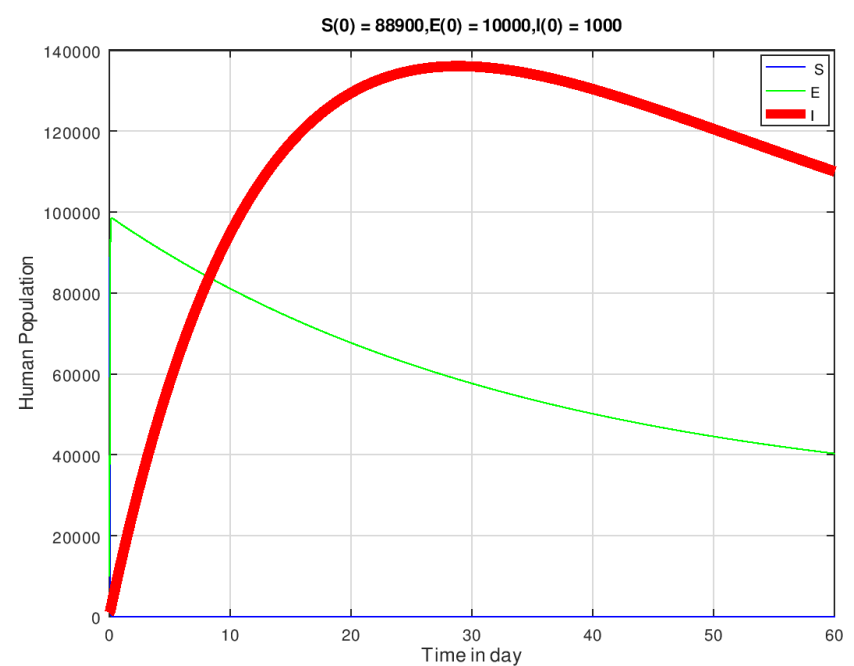

Figure 5. Numerical Simulation of the model with $K 1=0.008, K 2=0.005$, $P 1=0.19$ and $P 2=0.09$

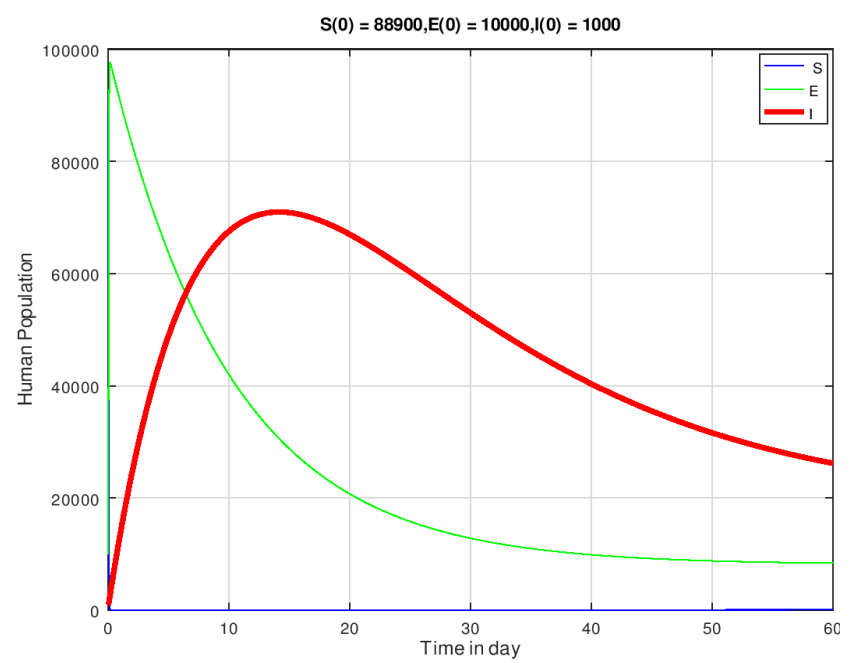

Figure 6. Simulation of the Model with $K_{1}=0.008, K_{2}=0.005, P_{I}=0.12$ and $P_{2}=0.09$.

In Figure 7 the susceptible, exposed and infected human population plotted vs time. High level of self isolation measures on exposed and infected population leads to reduce the exposed and infected human populations gradually with time within few weeks. At the same time the susceptible human populations decrease slowly and increases after weeks because of those strong self isolation measures of exposed and infected human populations.

In Figure 8 the exposed and infected human population increases slowly in the first few weeks and decrease slowly with increasing time. The susceptible human population decreases very slowly in the first weeks and increases after a weeks because of those strong self isolation measure on exposed and infected human population. Since $\mathrm{K}_{1}$ is changed from 0.00008 to 0.00005 and $\mathrm{K}_{2}$ is changed from $\mathrm{K}_{2}=$ 0.00005 to 0.000001 the infected and exposed human population decreases after few weeks. Since there is a strong self isolation measure on exposed and infected human population the susceptible human populations decreases very slowly and increases after a few weeks.

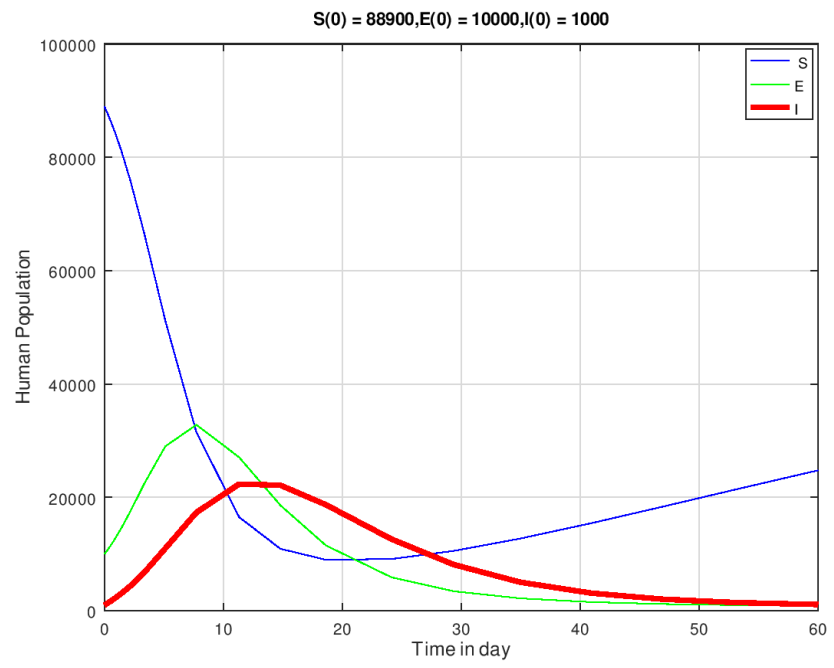

Figure 7. Numerical Simulation of the model with $P_{1}=0.009, P_{2}=0.005$, $K_{1}=0.00008$ and $K_{2}=0.00005$.

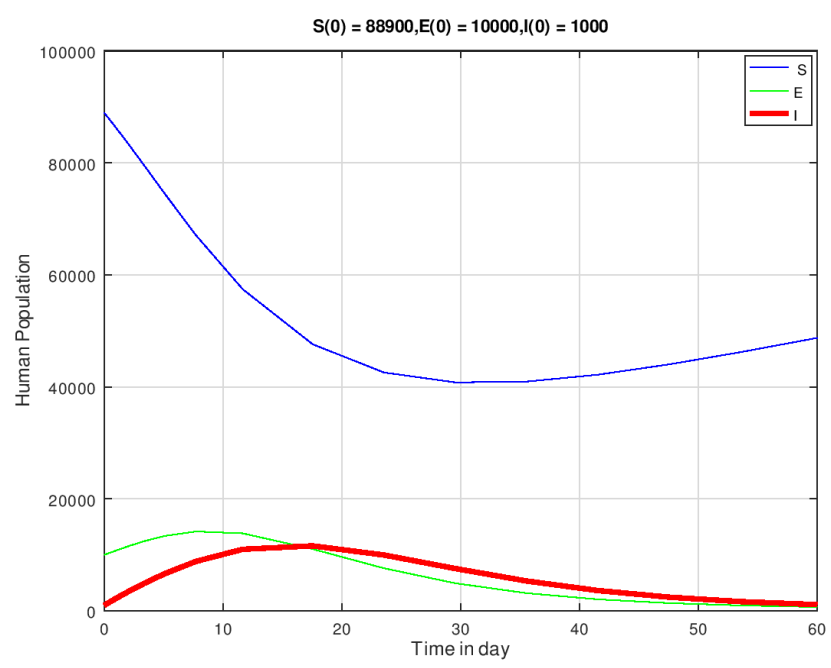

Figure 8. Simulation of the model with $P_{1}=0.009, P_{2}=0.005, K_{1}=$ 0.00005 and $K_{2}=0.000001$.

\section{Conclusion}

The models published and listed as a reference of our 
paper thus far have not taken into account the role of exposed and infected immigrants as well as the role of self isolation from the virus in the transmission of COVID-19. In the present study we have been proposed a mathematical model to investigate the on-going novel corona virus Covid-19 pandemic by considering Exposed and infected immigrants and self isolating techniques. It is found that the main reasons of successfully controlling and eliminating disease improving of self-protecting ability of susceptible, isolating all the close contacts, where asymptomatic cases or exposed cases are all belong to close contacts. And it tells us that quickly finding and self isolation of exposed (asymptomatic cases) can effectively control the spread of disease. The parameters $K_{1}$ and $\mathrm{K}_{2}$ have a bigger influence on the final size of accumulated confirmed cases, and this is consistent with the sensitivity analysis results of $R_{0}$. As well the parameters $\mathrm{P}_{1}$ and $\mathrm{P}_{2}$ have a significant role on the spread of covid-19 and $R_{0}$. If the exposed and infected human populations isolate themselves from contact to susceptible human population the spread of the virus will ends with in short period of time. As a result, it is recommended to the responsible body to isolate asymptomatic cases and practice home to home check up, as well as the responsible body should take measures on human mobility during the pandemic. We have defined the basic reproductive number $R_{0}$ which provides the expected number of new infections from one infectious individual over the duration of the infectious period given that all other members of the population are susceptible. We also showed that the existence of equilibrium points. Numerical simulation of the model shows the dynamic properties of human compartments versus time and the stabilities of the equilibrium points. We can observe from the numerical simulations that the exposed and infected human population increases with high values of the self isolation measures and with rates of exposed and infected immigrants. This shows that human mobility increases the dynamics and spread of covid-19 pandemic.

\section{References}

[1] Reza Sameni, Grenoble. 19 May 2020. Mathematical Modeling of Epidemic Diseases; A Case Study of the COVID-19 Coronavirus. https://arxiv.org/abs/2003.11371. 19 May 2020.

[2] Chay Y., Jin Wang. A mathematical model for the novel corona virus pandemic in Wuhan, China. Mathematical Biosciences and Engineering. 11 March 2020.

[3] Zhi-Qiang, et al. Modeling the Transmission of Middle East Respiratory Syndrome Corona Virus in the Republic of Korea. PLOSONE|DOI: 10.1371/journal.pone.0144778. December 21,2015 .

[4] P. van den Driessche, James Watmough. Reproduction numbers and sub-threshold endemic equilibria for compartmental models of disease transmission. Mathematical Biosciences.

[5] Novel Coronavirus (2019-nCoV) Situation Report. World Health Organization, 2020.
[6] B C K Choi, A W P Pak. A simple approximate mathematical model to predict the number of severe acute respiratory syndrome cases and deaths. J Epidemiol Community Health.

[7] Enahoro Iboi, et. al. Mathematical Modeling and Analysis of COVID-19 pandemic in Nigeria. https://doi.org/10.1101/2020.05.22.20110387. May 22, 2020.

[8] Meir Shillor, Nofe Al-Asuoad. Mathematical model and simulations of MERS outbreak: Predictions and implications for control measures. ResearchGate. January 2017.

[9] Lisa E. Gralinskiand Vineet D. Menacher. Return of the Coronavirus: 2019-nCoV. Viruses 2020, 12, 135; doi: 10.3390/v12020135. 24 January 2020.

[10] Seongwoo Yang et. al. Middle East respiratory syndrome risk perception among students at a university in South Korea, 2015. American Journal of Infection Control.

[11] Tianmu Chen, et. al. A mathematical model for simulating the transmission of Wuhan novel Coronavirus. http://dx.doi.org/10.1101/2020.01.19.911669doi. January 19, 2020 .

[12] Demsis Dejene, Purnachandra Rao Koy. Population Dynamics of Dogs Subjected To Rabies Disease. IOSR Journal of Mathematics (IOSR-JM).

[13] Wendi Wang. Backward Bifurcation of an epidemic model with treatment. Mathematical Bioscience. 8 February 2006.

[14] Faical Ndairouaet. al. Mathematical Modeling of COVID-19 Transmission Dynamics with a Case Study of Wuhan. Center for Research and Development in Mathematics and Applications.

[15] Benny Yong, and Livia Owen. Dynamical transmission model of MERS-CoV in two area. https://doi.org/10.1063/1.4942993 Published Online: 29 February 2016.

[16] Molalegn Ayana, Purnachandra Rao Koya. The Impact of Infective Immigrants on the Spread and Dynamics of Zika Virus. American Journal of Applied Mathematics. November 5, 2017.

[17] Natsuko Imai. et. al. Transmissibility of 2019-nCoV. WHO Collaborating Centre for Infectious Disease Modelling, MRC Centre for Global Infectious Disease Analysis, J-IDEA, Imperial College London, UK. 22/1/20-24/1/20.

[18] Ning Wang, et. al. An evaluation of mathematical models for the outbreak of COVID-19. Precision Clinical Medicine. doi: $10.1093 / \mathrm{pcmedi} / \mathrm{pbaa} 016$.

[19] D. Pal1; D. Ghosh, P. K. Santra, G. S. Mahapatra. Mathematical Analysis of a COVID-19 Epidemic Model by using Data Driven Epidemiological Parameters of Diseases Spread in India. https://doi.org/10.1101/2020.04.25.20079111doi. April 29, 2020.

[20] Alexander Okhuese Victor. Mathematical predictions for covid-19 as a global pandemic. ResearchGate. https://www.researchgate.net/publication/339944210. 31 March 2020.

[21] Alex Arenas, et. al. A mathematical model for the spatial temporal epidemic spreading of COVID19. Harvard Medical School \& Brigham and Women's Hospital, Boston MA 02115 , US. January 2020. 